\title{
Framing the figure: Mental rotation revisited in light of cognitive strategies
}

\author{
A. Reyyan Bilge ${ }^{1} \cdot$ Holly A. Taylor ${ }^{2}$
}

Published online: 6 September 2016

(C) Psychonomic Society, Inc. 2016

\begin{abstract}
The mental rotation literature commonly reports a sex difference, almost always favoring men. Two strategies have been proposed in the literature to account for this difference: holistic and piecemeal. However, there is great variability in rotation performance suggesting other possible contributing factors. This study investigated the effects of stimuli characteristics and habitual spatial thinking on mental rotation performance. In three experiments, participants completed a mental rotation task with two modifications: (1) 3-D figures were presented with their cut versions to promote piecemeal strategy, and (2) block figures were either presented within a frame or none, in light of reference framework model or perceptual grouping model. Overall, whole figures generated faster responses than cut figures and this was more pronounced with greater angular disparity. Shape or the presence of a frame affected rotation performance. Having a frame seemed to impede overall rotation, especially for cut figures, supporting an object-based reference frame. However, a 3-D frame did not have the same effect, possibly suggesting the unitary configuration idea. Men rotated more accurately than women, but still sex was not as robust as it was suggested in the literature. Interestingly, there were similarities between possible strategy use and habitual spatial thinking. People who were categorized as having landmark-centered representations and who preferred verbal directions showed a pattern suggesting the use of a piecemeal strategy, and survey-
\end{abstract}

A. Reyyan Bilge

reyyanbilge@sehir.edu.tr

1 Psychology Department, Istanbul Sehir University, Kusbakisi Cad. No: 2734662 Altunizade-Uskudar, Istanbul, Turkey

2 Department of Psychology, Tufts University, Medford, MA, USA centered representations and map preference seemed to reflect a holistic strategy.

Keywords Mental rotation - Cognitive strategies $\cdot$ Framing · Habitual spatial thinking

\section{Introduction}

Dave and Judy just moved to a new apartment. A delivery guy haphazardly places the parts of their new sectional sofa across the living room. Both Dave and Judy think about how to best arrange the sectional. Although they independently decide on the same location and configuration, Dave makes his decision more quickly. Talking about how they decided where it should go, they realize they used different approaches. While Dave re-arranged the sectional by thinking about it as a whole, Judy mentally manipulated the pieces one by one, potentially explaining why she contemplated the solution longer. Why did they adopt different strategies? Is it because Dave is a man and Judy is a woman? Or do other factors such as the walls of the room, or the way they are accustomed to think about space contribute to their cognitive strategy differences? The current research examined factors affecting cognitive strategies used for mental rotation.

We constantly process spatial information, e.g., moving without bumping into the furniture, knowing the direction between home and work, or fitting suitcases into a car trunk. Although we all engage in spatial processing, some people do so better than others. Individual differences in spatial ability have long captured researchers' attention, particularly sex differences (Collins \& Kimura, 1997; Kail, Carter, \& Pellegrino, 1979; Lippa, Collaer, \& Peters, 2010; Miller \& Halpern, 2014; Reilly, Neumann, \& Andrews, 2016), yet conclusions have not been consistent and questions remain (Voyer, Voyer, \& 
Bryden, 1995; Uecker \& Obrzut, 1993). However, sex is not the only factor that has shown a relationship to mental rotation. Spatial cognitive style (Pazzaglia \& DeBeni, 2001, 2006), stimuli characteristics, such as complexity (e.g., Bethell-Fox \& Shepard, 1988; Doyle \& Voyer, 2013; Doyle, Voyer, \& Lesmana, 2015; Folk \& Luce, 1987), dimensionality (e.g., Bauer \& Jolicoeur, 1996; Hampson, Levy-Cooperman, \& Korman, 2014; JansenOsmann \& Heil, 2007), or occlusion (Alexander \& Evardone, 2008; Doyle \& Voyer, 2013; Voyer \& Doyle, 2010; Voyer \& Hou, 2006), and instructions (e.g., Sharps, Price, \& Williams, 1994) can also affect mental rotation. The present work explores the sole and combined effects of stimuli characteristics, such as framing and figure consistency, and individual differences, such as habitual spatial thinking, on mental rotation performance. We begin by explaining the Mental Rotation task and how possible cognitive strategies might affect its performance. We go on to discuss factors, such as stimulus characteristics and habitual spatial thinking that could affect rotation performance.

\section{Mental rotation task}

In classic mental rotation experiments (e.g., Shepard \& Metzler, 1971), participants decide whether two figures are the same or mirror images of one another. The time to do so shows a linear relationship between the two figures' initial angular disparity and both response time (e.g., Shepard \& Metzler, 1971) and accuracy (e.g., Butler et al., 2006), an effect known as the Angular Disparity Effect (ADE). The $\mathrm{ADE}$ has been shown with psychometric multiple-choice tests (Alexander \& Evardone, 2008; Doyle \& Voyer, 2013; Vandenberg \& Kuse, 1978; Voyer \& Hou, 2006) and computerized Mental Rotation tests (Bryden, George, \& Inch, 1990; Doyle, Voyer, \& Lesmana, 2015). Based on this finding, researchers hypothesize that participants mentally rotate one figure to align with the other. Note, however, that recent work from Tufts' Spatial Cognition Lab (Gardony, Taylor, \& Brunye, 2014) suggests that rotation may not be to alignment, but to an angular disparity affording successful comparison. In actuality, this mental rotation process involves several steps (Cooper \& Shepard, 1973; Halpern \& Collaer, 2005; Just \& Carpenter, 1976). A person first encodes both stimuli then mentally rotates one to align with the other. Once aligned, the person compares the figures, deciding whether they are the same and then responds. Regression data showing the relationship between angular disparity and response accounts for the different mental rotation steps (Kail et al., 1979; Lohman, 1986); the slope reflects mental rotation rate, and the intercept includes encoding, comparison, and response processes. Eye movement data also suggest these steps (Freedman \& Rovegno, 1981; Just \& Carpenter, 1976).

Sex differences in mental rotation have received significant research attention. Not uncommonly, men mentally rotate faster than women (Linn \& Petersen, 1985; Lippa, Collaer, \& Peters, 2010; Maccoby \& Jacklin, 1974; Masters \& Sanders, 1993; Peters, 2005; Resnick, 1993; Richardson, 1994; Voyer, Voyer, \& Bryden, 1995) although accuracy is often comparable (Goldstein, Haldane, \& Mitchell, 1990; Kail et al., 1979; Voyer \& Bryden, 1990). In reality this male advantage may be more ethereal. Some studies have found no sex difference (Butler et al., 2006; Hugdahl, Thomsen, \& Ersland, 2006; Jansen-Osmann \& Heil, 2007; Jordan, Schadow, Wuestenberg, Heinz, Peters, \& Jancke, 2002; Peters, 2005; Voyer \& Bryden, 1990), especially when instructions emphasized general rather than spatial thinking (Scali, Brownlow, \& Hicks, 2000) or when the stimuli were haptically or visually experienced replicas of 3-D figures rather than drawings (Robert \& Chevrier, 2003). In other studies women were faster than men (Moè, 2009; Thomsen, Hugdahl, Ersland, Barndon, Lundervold, \& Smievoll, 2000; Uecker \& Obrzut, 1993). This variability in mental rotation sex differences suggests that other factors may contribute to strategy use, including aspects of the Mental Rotation task (e.g., stimuli characteristics) and/or other individual differences (e.g., habitual spatial thinking).

\section{Possible factors leading to differential mental rotation performance}

Strategies in mental rotation Different data patterns have been used to explain sex-linked cognitive strategies for mental rotation: response times slowed down as a function of angular disparity suggesting complexity of the stimuli affected rotation, and further made the difference between sexes more observable (Gluck \& Fitting, 2003; Halpern, Benbow, Geary, Gur, Hyde, \& Gernsbacher, 2007; Kail et al., 1979). Women also displayed a positively skewed response distribution, compared to men's normal distribution (Kail et al., 1979; Jordan et al., 2002; Lohman, 1986). Both the distribution and the slope difference suggest strategy differences.

Two mental rotation strategies have been proposed in the literature: holistic vs. piecemeal (Kail et al., 1979; Maccoby \& Jacklin, 1974; Strasser, Koller, Strauß, Csisinko, Kaufmann, \& Glück, 2010) or imagistic versus analytic (Heil \& JansenOsmann, 2008; Lawton, 2010; Pezaris \& Casey, 1991). We will adhere to the use of holistic and piecemeal strategies henceforth since the terms fit the conditions used in the current study. With holistic processing, the figure is rotated as a whole; with piecemeal, it is broken down into parts that are rotated separately (Geiser, Lehmann, \& Eid, 2006; Robertson \& Palmer, 1983; Yuille \& Steiger, 1982). Specifically, holistic strategies involve analog spatial processing and piecemeal 
strategies rely on feature-by-feature non-spatial comparison. Hemispheric laterality observed in mental rotation further supports these two strategies (e.g., Hugdahl et al., 2006).

Stimuli characteristics Naylor, Taylor, and Cross (1998) created stimuli to test holistic versus piecemeal strategies. Participants viewed 3-D cube figures that were either whole objects, akin to Shepard and Metzler's (1971) original figures, or the same figures cut in half to facilitate a piecemeal strategy. They found no sex difference, overall, but an interaction between sex and whole/cut objects that suggested strategy differences. As predicted, women responded similarly to cut and whole objects. Men's data for cut objects matched that of women, but men differed in their whole object rotation. This pattern suggested that women consistently took a piecemeal approach whereas men sought an efficient strategy for the stimuli at hand (Naylor, Taylor \& Cross, 1998). So, if mental rotation performance differences relate to strategy choices, stimuli promoting either piecemeal or holistic processing should interact with the strategy used.

Frame of reference is vital in spatial information processing (e.g., Levinson, 1996) and research showed that surrounding geometry affects the spatial representation (Kelly, McNamara, Bodenheimer, Carr, \& Rieser, 2008; Kelly \& McNamara, 2008; Mou, Zhang, \& McNamara, 2009; Mou, Zhao, \& McNamara, 2007; Wang \& Spelke, 2000). Gestalt perception, and perceptual grouping (Rock, 1990), specifically, suggests that a frame would emphasize an object's unitary structure, thus predicting more effective rotation with a frame. In contrast, the Multiple Systems Framework (Zacks \& Michelon, 2005) suggests that mental rotation engages object-based transformations. An object's reference frame moves relative to the observer. This theory predicts more effective rotation without a frame, because the frame imposes an environmentbased reference on an object-based transformation. Thus, a frame should impede performance.

How a frame affects mental rotation may also interact with stimulus consistency (whole vs. cut). With perceptual grouping theory (Rock, 1990), frame might force unity more so for whole figures and holistic processing, but might impede cut figure rotation. With the Multiple Systems Framework a piecemeal strategy, promoted by cut stimuli, might engage environment-based references to track piece-by-piece rotation (Zacks \& Michelon, 2005). This would suggest that a frame should hinder whole object rotation and would either not impact or might even help cut figure rotation.

Habits and preferences in spatial processing Just as with mental rotation, people adopt habitual preferences and strategies for processing spatial information at a greater scale. For example, when learning a new environment, one person may seek out a map while another may prefer to explore or get a verbal description. When thinking about a known environment, one person may view it as if from above (survey representation), someone else may imagine taking a route through the environment (route representation), and a third person may think about the various landmarks in the environment (landmark representation). The root of these habits and preferences has not been systematically explored, but they seem to affect other spatial processing.

Pazzaglia and De Beni (2001, 2006) developed and used a Questionnaire on Spatial Representation, which includes questions designed to understand whether an individual habitually uses a survey, route or landmark-centered approach when thinking about known environments or learning new places. They then related one's habitual approach to mental rotation. Overall, individuals, both men and women, who had survey-based representational preferences scored better on mental rotation compared to individuals who habitually took a landmark or route-centered approach (Pazzaglia \& De Beni, 2001). Thus, one's habitual spatial processing; i.e., the way they habitually represent spatial information, appears to impact spatial processing more generally. One's preference for how he/she learns a new environment (maps, exploration, verbal description) may have a similar effect.

\section{Current research}

The current research, in three experiments, investigated various influences on mental rotation performance and strategies. Overall, we expected to replicate the standard ADE in mental rotation research (e.g., Shepard \& Metzler, 1971). Experiment 1 employed a standard 3-D rotation task, but with modified stimuli. The first modification (whole vs. cut objects) encouraged a holistic or piecemeal approach. The second modification (unframed vs. framed) allowed for standard reference frame use or introduced a salient alternative reference frame. In Experiment 2, we further investigated the rotation performance on reference frame use, introducing a circular frame in addition to no frame and the rectangular frame. A circular frame provides a salient basis for comparison, but without the axes that define a reference frame. Experiment 3 explored how the same stimulus modifications impacted the rotation of a 3-D figure within a 3-D frame, again using whole and cut figures. Overall, rotation performance for strategy-consistent stimuli should reflect the pattern indicative of that strategy. All experiments also explored sex differences and habitual spatial processing differences in the form of spatial representation (Pazzaglia \& De Beni, 2001) and preference for learning spatial information.

\section{Experiment 1}

In mental rotation tasks, stimuli generally consist of whole objects. People imagine these rotating as a solid unit (holistic 
strategy) or cognitively sub-divide them (piecemeal strategy). These different strategies are evident in response time (RT) patterns, such as overall faster performance with a holistic strategy, and greater increase in RT as a function of the angular difference with a piecemeal strategy. Experiment 1 explored how stimulus characteristics that create affordances for either a holistic or piecemeal strategy affect rotation performance.

We modified the standard Shepard and Metzler (1971) type figures to encourage a piecemeal strategy (cut objects) and test strategy use with frames surrounding each object. While stimulus differences, such as those manipulated in Experiment 1, might encourage a particular mental rotation strategy, people have habitual cognitive approaches to spatial tasks. To examine how these habitual spatial approaches affect rotation, we included individual difference measures that illuminate them.

\section{Method}

\section{Participants}

Ninety-two Tufts undergraduates, 40 women and 52 men, participated for partial course credit.

\section{Materials}

Mental rotation stimuli Stimuli consisted of object pairs defined by three factors; Angular Disparity $\left(0^{\circ}, 30^{\circ}, 60^{\circ}, 90^{\circ}\right.$, $120^{\circ}, 150^{\circ}$, and $180^{\circ}$ ), Figure Consistency (whole, cut), and Framing (framed, unframed). Stimuli consisted of three 3-D Shepard and Metzler (1971) type figures created with CAD software. Each figure included ten or eleven cubes with 90degree angular projections into 3-D space. These 3-D block figures comprised the whole figures. We created cut figures by dividing the whole figures into two parts (one half with five cubes and the other with five or six) and separating the halves by 0.75 inches (Naylor et al., 1998). In total, there were 63 whole and 63 cut figures, totaling to 126 trials per experiment. We also created a mirror-version of each figure, totaling to 63 same and 63 mirror images. For all figures, we created framed stimuli by adding a rectangular frame, approximately $6 \times 4$ in., surrounding each whole figure and both halves of each cut figure. In total, there were 126 framed and 126 unframed figures. This way, participants were tested on each angular disparity 14 times; seven in whole, seven in cut blocks; and seven in unframed and seven in framed conditions.

Stimulus pairs consisted of two whole or two cut figures (Fig. 1) that were either the same or different (mirror image) from one another. A vertical line separated the two figures in a pair. Pairs of objects (whole and cut) differed in angular rotation by 30 -degree-increments, yielding seven rotations: $0^{0}$, $30^{\circ}, 60,90^{\circ}, 120^{\circ}, 150^{\circ}$, and $180^{\circ}$, and included rotations along $\mathrm{X}$-, Y-, and Z-axes.

Questionnaires Questionnaires gathered information on factors implicated in differential habitual spatial thinking. The Individual Differences questionnaire asks participants about gender, familial sinistrality, self-rated sense of direction, and preference for spatial information format (map or verbal directions). The Mental Representation questionnaire (Pazzaglia \& De Beni, 2001) assesses spatial processing habits, including sense of direction and cognitive approaches to spatial processing. There are 17 questions in total and an examplary question would be "When you are in your home-town or city, do you naturally know cardinal points? That is, do you know where North, South, East, and West are?" Most of the questions ask participants to rate their answers on a Likert-type scale, from 1 to 5 where 1 indicates "not at all" and 5 indicates "very much." Through coding the questions in a certain way (Pazzaglia \& De Beni, 2001, 2006), a person can be identified as habitually using a survey-centered versus landmark-centered representation.

\section{Design}

In Experiment 1 characteristics of stimuli were manipulated: (1) Participants (46 participants) either received framed stimuli or they (46 participants) received unframed stimuli). (2) Within the framing condition, participants either received whole figures or their cut versions.

The questionnaires that participants completed allowed us to categorize them by: (1) sex, (2) preference when learning new environments (maps or verbal directions), and (3) mental representations tendency (survey or landmark). Thus, the present study used a 2 (framed vs. unframed, between participant) $\times 2$ (whole vs. cut, within participant) $\times 2$ (individual factors: sex, preference, or mental representation, between participant) mixed design. The dependent variable was performance (rotation rate and accuracy) on the Mental Rotation test.

\section{Procedure}

The experiment took place in Tufts University's Spatial Cognition Lab. After consenting to take part, participants sat in front of a computer running Superlab ${ }^{\mathrm{TM}}$ software.

For the Mental Rotation trials, participants read instructions describing our two-part response paradigm. With this paradigm, participants, on each trial, first view a fixation cross and then see the mental rotation pair. They are to hit the space bar when they know whether the figures are the same or mirror images. This will be referred to as the know response. After hitting the space bar the same pair appears and participants respond same or different, using designated keys. This will be referred to as the answer response. We employed this two-part response paradigm to separate RT for mental rotation from RT 


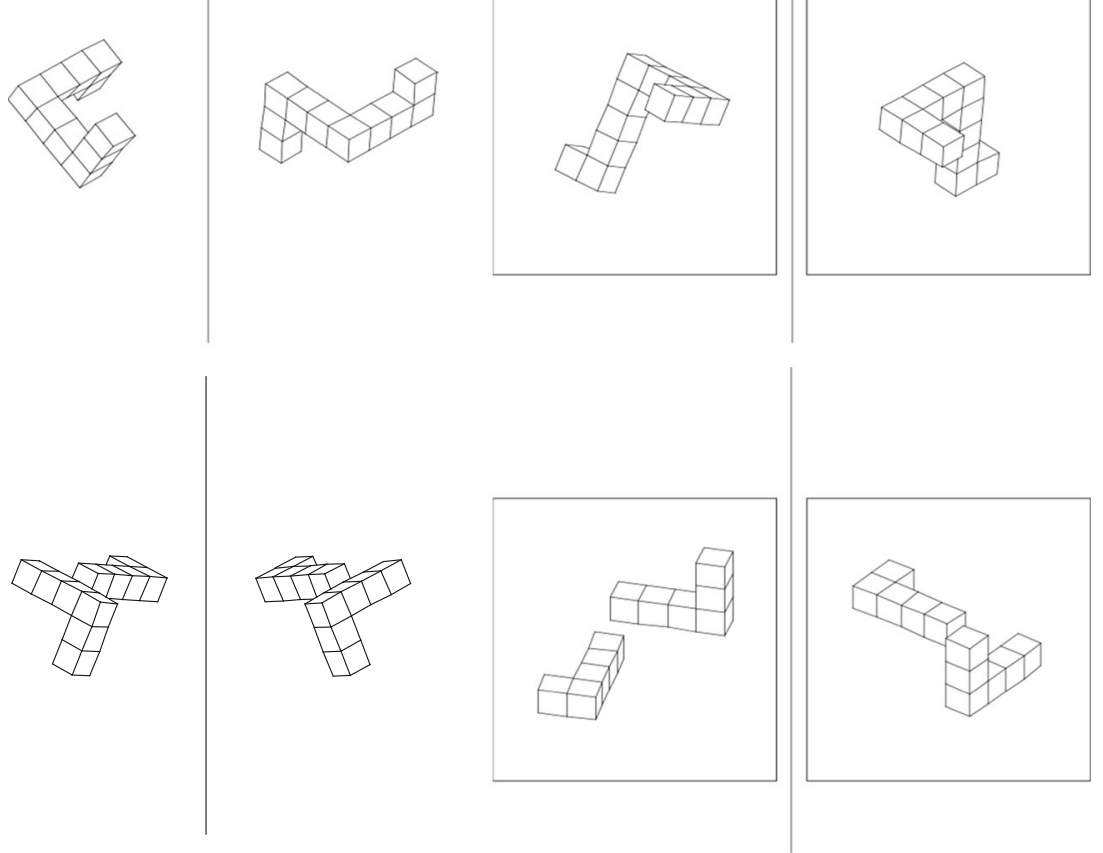

Fig. 1 (a) (top left) A pair of unframed whole figures, while (b) (top right) shows a pair of framed cut figures. (c) (above left) An unframed cut pair and (d) (above right) shows framed cut stimuli

for other aspects of choice reaction time tasks (Donders, 1969). Participants progressed through trials at their own pace. The experiment was divided into two blocks (whole figures and cut figures) that were randomly counterbalanced across participants. Each block contained 63 trials, yielding 126 total trials.

After completing both experimental blocks, all participants completed the Individual Differences questionnaire. Participants in the framed group completed the study later and they additionally completed the Mental Representation questionnaire along with the Individual Differences questionnaire.

\section{Results}

\section{Coding and analysis}

Data from seven people were eliminated for not following instructions properly. Moreover, one participant had lower than $50 \%$ overall accuracy rate, and two participants were outliers and their data were not included in the analysis. Analyzed data included RT to the know response and accuracy of answer responses. Given the high response variability, data were trimmed by eliminating each participant's highest and lowest know RT responses (Bush, Hess, \& Wolford, 1993).

Statistical assessment used mixed-factor ANOVA with a 7 (Angular Disparity: $\left.0^{\circ}, 30^{\circ}, 60^{\circ}, 90^{\circ}, 120^{\circ}, 150^{\circ}, 180^{\circ}\right) \times 2$ (Figure Consistency: whole, cut) $\times 2$ (Framing: framed, unframed). Angular Disparity and Figure Consistency served as within-participant factors and Framing as a betweenparticipant factor. For brevity's sake, we first report results of this omnibus ANOVA, which is the main focus of interest in this study. We ran another mixed-factor ANOVA with a 7 (Angular Disparity) $\times 2$ (Figure Consistency) $\times 2$ (Sex: men, women) design. We then replaced factors of habitual spatial thinking (Preference and Mental Representation) with Sex. We did not run all these factors concurrently in order to conserve power. Considering that the other main focus of interest is the interaction between habitual spatial thinking on strategy-consistent stimuli in mental rotation, we included Preference and Mental Representation into the analysis, one by one. Sections reporting sex differences and habitual spatial thinking results only relate additional reports beyond the omnibus ANOVA.

Data were analyzed using a mixed design ANOVA and Mauchly's test indicated that the assumption of sphericity has been violated for Angular Disparity for RT data, $X^{2}(20)=130$, $p<.001$, and for the accuracy data, $X^{2}(20)=51.4, p<.001$. Therefore, degrees of freedom were corrected using Greenhouse-Geisser estimates of sphericity $(\varepsilon=.65)$ for RT, and Huynh-Feldt correction $(\varepsilon=.87)$ for accuracy. Assumption of sphericity has also been violated for Angular Disparity* Figure Consistency interaction; RT data, $X^{2}(20)=58.3, p<$ .001 , and accuracy data, $X^{2}(20)=41.1, p=.004$. On both RT and accuracy, degrees of freedom were corrected using HuynhFeldt estimates of sphericity; $\varepsilon=.92, \varepsilon=.94$, respectively. 


\section{Mental rotation performance}

The mean RT for the experiment is 6,240 ms and overall accuracy is $77.9 \%$. Results showed the classic ADE with increased RT, $F(3.89,350)=134, p<.001, \eta_{\mathrm{P}}{ }^{2}=.60$, (Fig. 2a), and decreased accuracy, $F(5.19,467)=56.23, p<.001, \eta_{\mathrm{P}}{ }^{2}=.39$ (Fig. 2b) as a function of Angular Disparity. The error bars represent standard errors for all the figures in the manuscript.

\section{Do strategy-consistent-stimuli affect mental rotation performance?}

The analysis showed an effect of Figure Consistency. Participants responded faster and more accurately to whole $\left(\mathrm{M}_{\mathrm{RT}}=5794 \mathrm{~ms}\right.$, $\mathrm{M}_{\mathrm{acc}}=82.5 \%$ accuracy $)$ than to cut objects $\left(\mathrm{M}_{\mathrm{RT}}=6,685 \mathrm{~ms}\right.$, $\left.\mathrm{M}_{\mathrm{acc}}=73.3 \%\right), F(1,90)=14.4, p<.001, \eta_{\mathrm{P}}{ }^{2}=.14$ for mean RT and $F(1,90)=53.9, p<.001, \eta_{\mathrm{P}}^{2}=.38$ for accuracy.

Figure Consistency interacted with Angular Disparity with both mean RT, $F(5.49,494)=7.88, p<.001, \eta_{\mathrm{P}}{ }^{2}=.080$, and mean accuracy, $F(5.66,509)=9.84, p<.001, \eta_{\mathrm{P}}{ }^{2}=.099$. For small $\left(30^{\circ}, 60^{\circ}\right)$ angular differences, participants rotated whole and cut figures equally fast. At greater angular disparities, they rotated whole figures faster (see Fig. 3a) and more accurately (Fig. 3b).

\section{Does framing stimuli affect mental rotation performance?}

Framing did not affect mental rotation performance by itself in rotation rate, $F(1,90)=2.24, p=.14$, or rotation accuracy, $F(1,90)=.022, p=.88$. However, it interacted with Figure Consistency for mean RT data, $F(1,90)=4.22, p=$ $.038, \eta_{\mathrm{P}}^{2}=.045$. When the figures were unframed, participants responded equally quickly to whole and cut figures, whereas when there was a frame around, cut figures generated slower responses (see Fig. 4).

There was also a three-way interaction between Frame $x$ Figure Consistency $\times$ Angular Disparity for mean RT data, $F(5.49,494)=2.87, p=.012, \eta_{\mathrm{P}}^{2}=.031$. This 3-way interaction is a further depiction of distinct slower response time to both framed and cut stimuli. For unframed stimuli (see Fig. 5a), participants responded similarly to whole and cut figures for $30^{\circ}, 60^{\circ}, 150^{\circ}$, and $180^{\circ}$. However, for framed stimuli (see Fig. 5b), they responded equally fast to whole and cut objects at smaller angular differences $\left(30^{\circ}, 60^{\circ}\right)$, but the difference was more evident for greater angular differences $\left(>60^{\circ}\right)$.

\section{Does sex of the participant affect mental rotation performance?}

Sex did not show a main effect for RT, $F(1,90)=2.96, p=.09$. However, women responded to Mental Rotation figures less accurately $\left(\mathrm{M}_{\mathrm{acc}}=73.1 \%\right), F(1,90)=17.57, p<.001, \eta_{\mathrm{P}}{ }^{2}=$ .16 than men $(\mathrm{Macc}=81.6 \%)$. Sex did not show any further interactions with Angular Disparity or Figure Consistency on either RT or accuracy data, all $F s<1.0$.

\section{Does habitual spatial thinking affect mental rotation performance?}

Spatial information preference (henceforth referred to as Preference) was categorized on participants' self-reports on learning spatial information-maps or verbal descriptions. Sixty-three people preferred maps, 26 people preferred verbal directions, and three preferred both when given spatial information. Those selecting "both" were not included in the analyses. In Preference, it is important to know the extent to which it overlaps with Sex (Table 1). Men (80.4\%) and women $(57.9 \%)$ preferred maps more than verbal descriptions, and men preferred map descriptions over verbal directions more so than women, $\mathrm{c}^{2}(1, N=89)=5.33, p<.05$. Importantly both sexes have representation in both preference groups.

Map preference participants responded more accurately (M $=81.4 \%)$ than verbal preference participants $(\mathrm{M}=70.1 \%)$, $F(1,87)=27.8, p<.001, \eta_{\mathrm{P}}{ }^{2}=.24$. The data also showed a Preference $\times$ Angular Disparity interaction for accuracy, $F(5.56,484)=5.57, p<.001, \eta_{\mathrm{P}}^{2}=.060$. Participants who preferred verbal directions responded less accurately than those with map preference over degrees of rotation (Fig. 6), and their
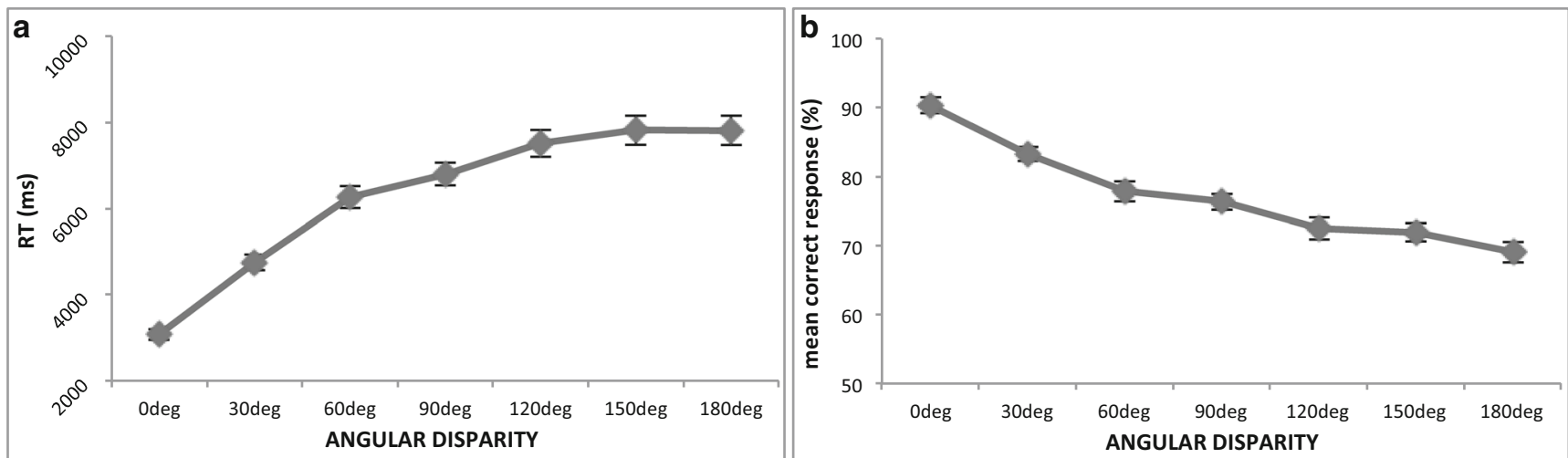

Fig. 2 Both Know RT (a; left) and accuracy (b; right) as a function of Angular Disparity 

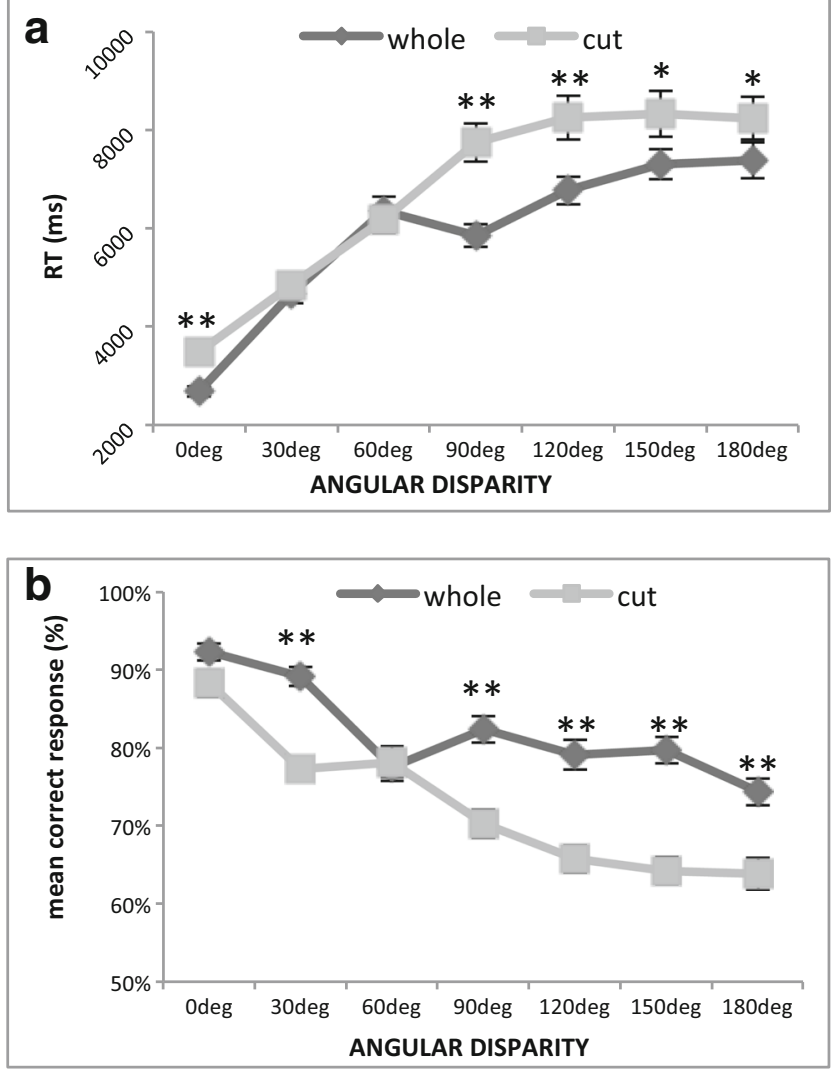

Fig. 3 Response times (a: top) and correct percentages (b: bottom) to whole and cut figures on degrees of rotation. Note: Paired t-test analyses were done to compare whole and cut figures at each degree of rotation. Results are depicted with asterisks. * denotes significance criterion of .05 $(\mathrm{p}<.05)$ for the corresponding degree of rotation and $* *$ denotes the significance criterion of $.001(\mathrm{p}<.001)$

responses varied across different angles while map preference showed a steady decline as a function of angular disparity.

Mental representation (henceforth referred to as Representation). The Representation questionnaire was only administered to participants later in the study and those people were the ones who were exposed to Framed stimuli. As with Preference, it is important to know the extent to which Sex and

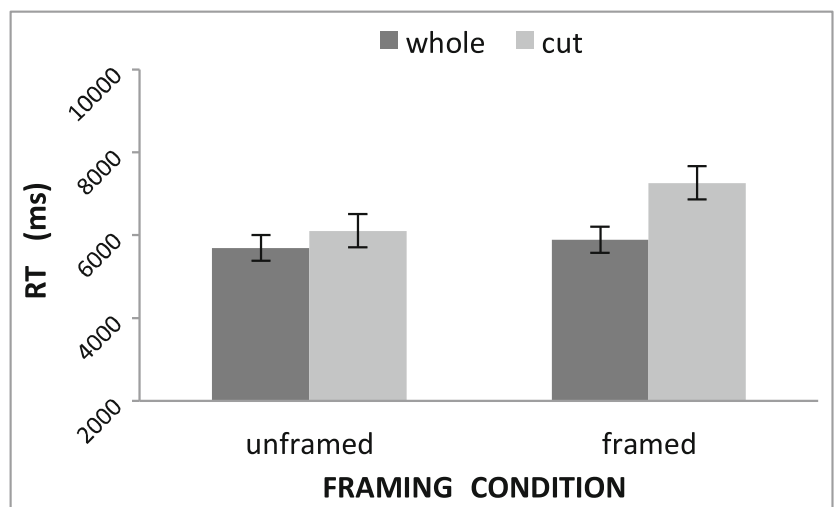

Fig. 4 Response rates to whole and cut figures when they are unframed or framed
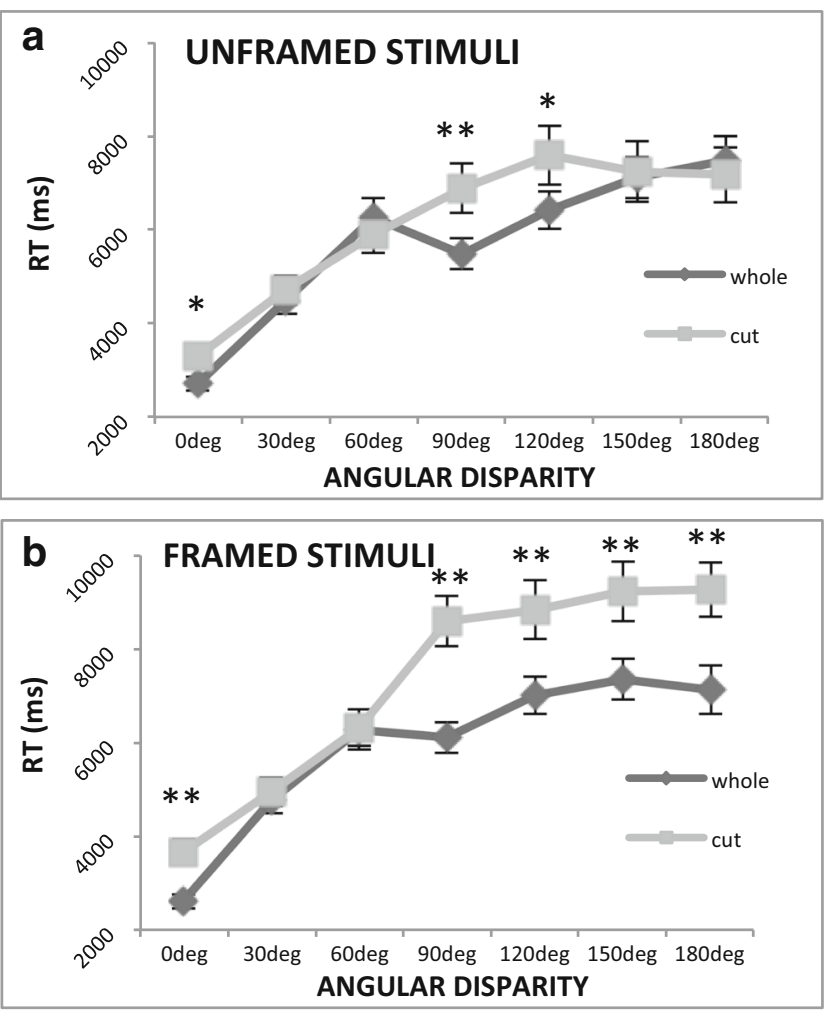

Fig. 5 Performance to whole and cut figures over degrees of rotation is shown as a function of frame. (a) (top) Mean response times for unframed stimuli and (b) (bottom) shows mean response times for framed stimuli. Paired t-test analyses were done to compare whole (darker line) and cut (lighter line) figures at each angular disparity, for both unframed and framed conditions. * significance at .05 level $(\mathrm{p}<.05), * *$ significance at .001 level $(\mathrm{p}<.001)$

Representation overlap (see Table 1). Men were categorized as having more survey-centered representations than women, who had more landmark-representations $\left(\mathrm{c}^{2}(1, N=89)=7.39, p<\right.$ $.05)$. Women showed habitual tendencies toward primarily using landmark-centered representations $(77.3 \%)$ and men primarily used survey-centered representations $(62.5 \%)$ and both sexes were represented in both landmark and survey groups.

Overall, survey-centered individuals responded faster (survey: 5,543 ms; landmark: 7,374 ms), $F(1,44)=7.20, p=.01$, $\eta_{\mathrm{P}}^{2}=.14$, and more accurately (survey: $81.8 \%$; landmark: $75.2 \%), F(1,44)=4.87, p=.033, \eta_{\mathrm{P}}^{2}=.10$. However, Representation data did not result in any interactions with Angular Disparity or Figure Consistency (all $F s<1.0$ ). especially after corrections according to sphericity assumptions.

\section{Discussion}

Consistent with findings within the literature, we showed the classic Angular Disparity Effect (ADE) in Mental Rotation task: participants decided whether a pair of 3-D figures were the same or different more slowly and less accurately as the angular disparity between the figures increased (Blough \& Slavin, 1987; 
Table 1 Sex ratios in reference to preference and representation

\begin{tabular}{|c|c|c|c|c|}
\hline & \multicolumn{2}{|c|}{ Preference } & \multicolumn{2}{|c|}{ Representation } \\
\hline & Verbal & Map & Landmark & Survey \\
\hline Women & 16 & 22 & 17 & 5 \\
\hline Men & 10 & 41 & 9 & 15 \\
\hline
\end{tabular}

Collins \& Kimura, 1997; Cooper, 1975; Heil \& JansenOsmann, 2008; Kail et al., 1979; Shepard \& Metzler, 1971). Experiment 1 results did not replicate the commonly reported sex finding with men outperforming women in rotation rate while men were still more accurate than women. Thus, our findings relating to performance on mental rotation did show a sex difference, but this difference was not as robust as the literature suggests (Collins \& Kimura, 1997; Linn \& Petersen, 1985; Masters \& Sanders, 1993; Voyer et al., 1995). The idea of other individual differences contributing to the difference in mental rotation performance becomes more important.

Current research extended previous mental rotation studies by introducing strategy-consistent stimuli (namely, cut versions of whole figures). To our knowledge, the combination of factors examined here is new to the literature. Stimulus characteristics, particularly whether the stimuli themselves are presented as whole or cut, contribute to response strategies. We predicted that having figures in pieces would promote piecemeal processing while whole figures would enable more holistic processing. Consistent with our prediction, participants rotated whole figures faster and more accurately. Moreover, they rotated cut figures more slowly and less accurately as angular disparity increased, a pattern suggested to account for strategy differences (Kail et al., 1979; Strasser, et al., 2010). Thus, although with caution, we can state that Experiment 1 was successful in using both whole and cut figures to reflect holistic and piecemeal strategies used in mental

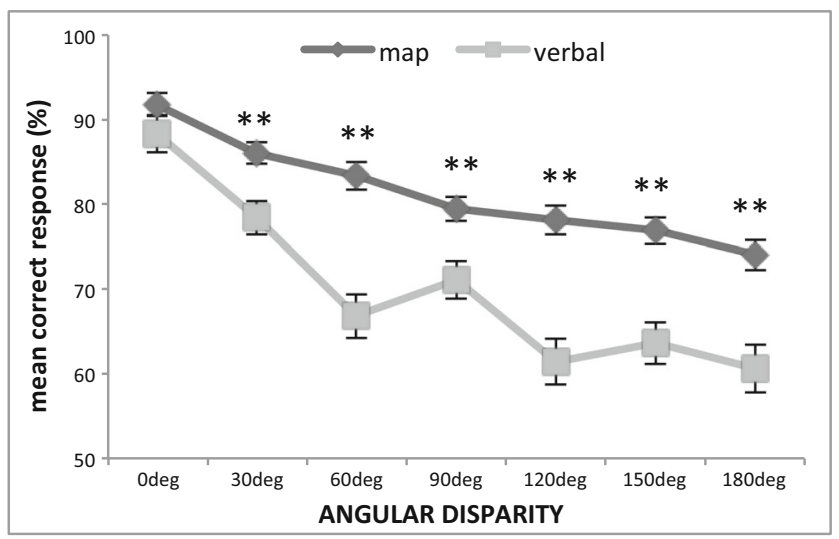

Fig. 6 Accuracy rates for people preferring maps and verbal directions over degrees of rotation. The dark line shows map preference while light line shows verbal preference. Paired t-test analyses depict the significant comparison for each degree of rotation. Both $*$ and $* *$ show significant results. * presents alpha level at $.05(\mathrm{p}<.05)$ and $* *$ marks a more stringent significance criteria $(\mathrm{p}<.001)$ rotation. Whole figures seemed to promote a holistic strategy and cut figures promoted a piecemeal strategy.

Experiment 1 also introduced frames around the figures. Framing the stimuli, overall, did not affect rotation performance. However, framing the cut figures seemed to hurt their processing speed. Responses to cut figures slowed down more as angular disparity increased. Frame seemed to heighten performance differences between whole and cut figures, especially for greater angular disparities, mimicking the cognitive strategy pattern. With framed stimuli, whole figure rotation mirrored holistic processing while cut figures reflected piecemeal approach. When there was no frame around the figures, however, no consistent pattern between whole and cut rotations was observed. These findings support the motivation behind stimulus manipulations showing that framing emphasized the unitary configuration of an object, especially for whole figures.

Whether a person prefers to receive spatial information via maps or verbal directions, and the way she represents that information (survey vs. landmark; Pazzaglia \& DeBeni, 2001) was hypothesized to reflect strategy differences in mental rotation. Rotation performance differed for both preference and representation factors; map preference was more accurate and became more accurate as angular disparity between the rotated pairs increased, mimicking the holistic strategy pattern suggested for men. Also, participants who relied more on survey-centered representations were more accurate and faster in mental rotation performance than people who used landmark-representations. Thus, habitual spatial thinking seems to affect rotation performance, and showing signs of possible strategy differences overriding sex differences. However, all these findings need to be evaluated with great caution. Participant numbers, especially after breaking down sexes into preference or representation styles, reduce the power to report the findings in confidence.

\section{Experiment 2}

In Experiment 1, surrounding 3-D figures with 2-D rectangular frames affected mental rotation performance. Experiment 2 used frames of different shapes (circular and rectangular), still 2-D, to explore the frame's role, either as emphasizing the unity of the object (circular and rectangular) or providing a reference frame for comparison (rectangular only). Different versions of the typical mental rotation paradigm use different shaped frames (Borst, Kievit, Thompson, \& Kosslyn, 2011; Hoyek, Collet, Fargier, \& Guillot, 2012; Kooshabeh \& Hegarty, 2010; Miller \& Halpern, 2014; Neuburger, Jansen, Heil, \& Quasier-Pohl, 2012; Vandenberg \& Kuse, 1978). In Experiment 2, we compared rotation performance of two frame types to un-framed figures in order to extensively test framing effects. And since the main focus was on frame types, we did not include cut versions of the stimuli. 


\section{Method}

\section{Participants}

Fifty-three Tufts University students (35 women, 18 men) participated for partial course credit. None had participated in the previous experiments.

\section{Materials}

Mental rotation stimuli Experiment 1's 3-D stimuli (only whole ones) were again used, combined with three frame types: no-frame, rectangular (Experiment 1) and circular. When present, frames surrounded each figure (Fig. 7). Stimuli were paired to have same (yet rotated) or mirrorreflection combinations with a vertical line dividing the pair. In total there were 126 trials for each frame type, yielding 378 trials. Of the 126 trials, 63 were the same and 63 were mirror reflections. Each angular difference was represented 18 times within a frame type.

Questionnaires The individual differences and spatial mental representation questionnaires used in Experiment 1 were again used here.

\section{Design}

In Experiment 2 stimuli were manipulated. In addition to the Framing conditions of Experiment 1, Experiment 2 also included circular frames.

Participants were categorized on sex, preference, and representation based on their self-reports. Thus, the present study was based on a 3 (circular-framed, rectangular-framed, unframed, within participant) $\times 2$ (individual factors: sex, preference, or mental representation, between participant) mixed design. The dependent variable was the performance on the Mental Rotation test.

\section{Procedure}

The procedure followed that of previous experiments. Participants sat in front of computers after consenting to partake in the experiment. Instructions preceded each of the three experimental blocks, defined by the frame types. Block order was counterbalanced across participants. On each trial participants first saw a fixation cross, followed by a pair of figures. Participants responded "same" or "different" by pressing designated keys. After the task participants completed both questionnaires.

\section{Results}

\section{Coding and analysis}

Dependent measures included accuracy and RT. Data were trimmed by eliminating each participant's highest and lowest RT at each angular disparity (Bush et al., 1993). Three participants' data were eliminated because they either did not follow instructions (2) or their accuracy was lower than $50 \%$ (1).

Repeated-measures analysis of variance (ANOVA) on mean percentage of correct response and RT data was conducted with 3 (Frame Type: unframed, rectangular, circular) $\times 7$ (Angular Disparity: $0^{\circ}, 30^{\circ}, 60^{\circ}, 90^{\circ}, 120^{\circ}$, $150^{\circ}$, and $\left.180^{\circ}\right) \times 2$ (Sex: men, women; or Preference: map, verbal directions; or Representation: survey landmark). Angular Disparity, and Frame Type were withinparticipant factors. Individual difference measures, including Sex, Preference, and Representation were betweenparticipant factors of primary interest. Sex and habitual thinking (preference, representation) were analyzed separately adding each to the omnibus ANOVA in order conserve power and effect sizes, as were done in Experiment 1. According to Mauchly's test, the assumption of sphericity was violated for Angular Disparity for RT data, $X^{2}$

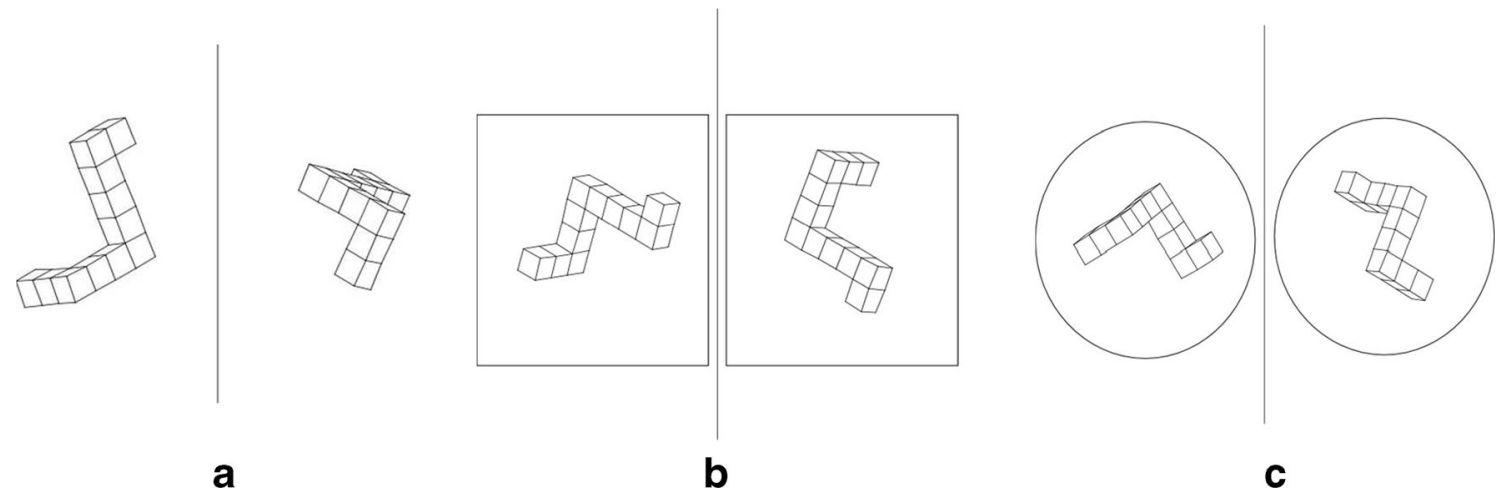

Fig. 7 Stimuli with different frame types: (a) unframed, (b) rectangular frame, and (c) circular frame 
$(20)=220, p<.001$, and for the accuracy data, $X^{2}(20)=$ $50.8, p<.001$. And degrees of freedom were corrected using Greenhouse-Geisser estimate for RT data of $(\varepsilon=$ $.30)$ and Huynh-Feldt for accuracy data, $(\varepsilon=.89)$ for accuracy. Angular Disparity* Frame Type interaction also violated the sphericity assumption, for RT data: $X^{2}(77)=$ $219, p<.001, \varepsilon=.60$ (Greenhouse-Geisser correction); and for accuracy data: $X^{2}(77)=103, p<.05, \varepsilon=.89$ (Huynh-Feldt correction).

\section{Mental rotation performance}

The mean RT for the experiment was $4,024 \mathrm{~ms}$ and the overall accuracy is $73.2 \%$. Results showed the ADE with increasing RT, $F(2.05,105)=91.9, p<.001, \eta_{\mathrm{P}}^{2}=.64$, and decreasing accuracy, $F(5.31,271)=57.1, p<.001$, $\eta_{\mathrm{P}}^{2}=.53$, with increasing angular disparity (see Fig. 8a and $b$ ).

\section{Does framing stimuli affect mental rotation performance?}

Frame Type also affected mental rotation performance only in rotation speed, $F(2,102)=3.62, p<.05, \eta_{\mathrm{P}}^{2}=.066$. Simple contrasts comparing frame types showed similar rotation performance with circular and rectangular framed stimuli $\left(\mathrm{M}_{\mathrm{cir}}=\right.$ 4,601.7 ms; $\mathrm{M}_{\mathrm{rec}}=4,362.9 \mathrm{~ms}$ ), however participants rotated unframed stimuli faster $\left(\mathrm{M}_{\mathrm{unfr}}=3,785.9 \mathrm{~ms}\right)$ than either frame type $(p ’ s<.05)$.

\section{Does sex of the participant affect mental rotation performance?}

Sex did not show any main effect for RT, $F(1,51)=.003, p=$ .96 , or accuracy data, $F(1,51)=2.99, p=.089$. However, there was a marginal Sex $\times$ Angular Disparity interaction with the accuracy data, $F(5.31,271)=2.17, p=.054, \eta_{\mathrm{P}}{ }^{2}=.041$. With increasing angular disparity, the performance gap between men and women slightly increased (Fig. 9).

\section{Does habitual spatial thinking affect mental rotation performance?}

Preference did not affect performance alone $(F=3.29)$. The distribution of Preference among sexes shows a tendency for both women $(74 \%)$ and men $(78 \%)$ to prefer maps (Table 2). Preference did not differ by Sex, $\mathrm{c}^{2}(1, N=53)=.078, p=.78$.

Rotation rate data showed a Preference $\times$ Angular Disparity interaction, $F(2.21,113)=5.59, p<.05, \eta_{\mathrm{P}}^{2}=$ .099 . The ADE was more pronounced for participants preferring verbal directions (Fig. 10).

The Preference $\times$ Angular Disparity interaction can be further qualified by marginally significant three-way interaction between Preference, Angular Disparity, and Frame Type, $F(7.36,375)=1.96, p=.057, \eta_{\mathrm{P}}^{2}=.037$. Mental rotation speed for participants preferring verbal directions differed as a function of both angular disparity and frame, showing the greatest $\mathrm{ADE}$ for circular frames followed by rectangular frames and least for no frame (see Fig. 11b). However, with respect to participants who preferred maps, they showed an ADE, but no effect of frame (see Fig. 11a). Accuracy data did not show any interactions (all $F_{S}<1.0$ ).

Representation Both men and women showed both habitual spatial representation approaches (landmark, and survey). Women were more inclined to use a landmark approach $(57 \%)$ and men relied more on a survey approach (56 \%). However, each category had representation from both sexes (see Table 2). Women and men did not show a difference in categorized representation style, $\left(c^{2}(1, N=53)=.769, p=\right.$ .380). Representation did not affect rotation performance either for mean RT data, $F(1,51)=1.74, p=.19$, or for accuracy data, $F(1,51)=.85, p=.36$.

\section{Discussion}

Overall, results showed the standard ADE (e.g., Collins \& Kimura, 1997; Shepard \& Metzler, 1971) with participants rotating 3-D figures more slowly and less accurately as the
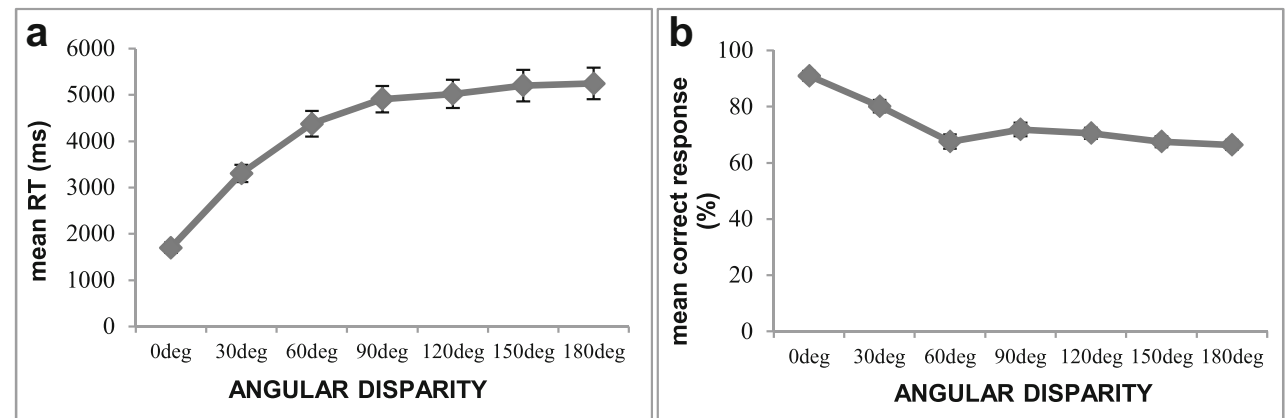

Fig. 8 (a) (left) shows mean reaction times and (b) (right) shows mean accuracy at each degree of rotation 


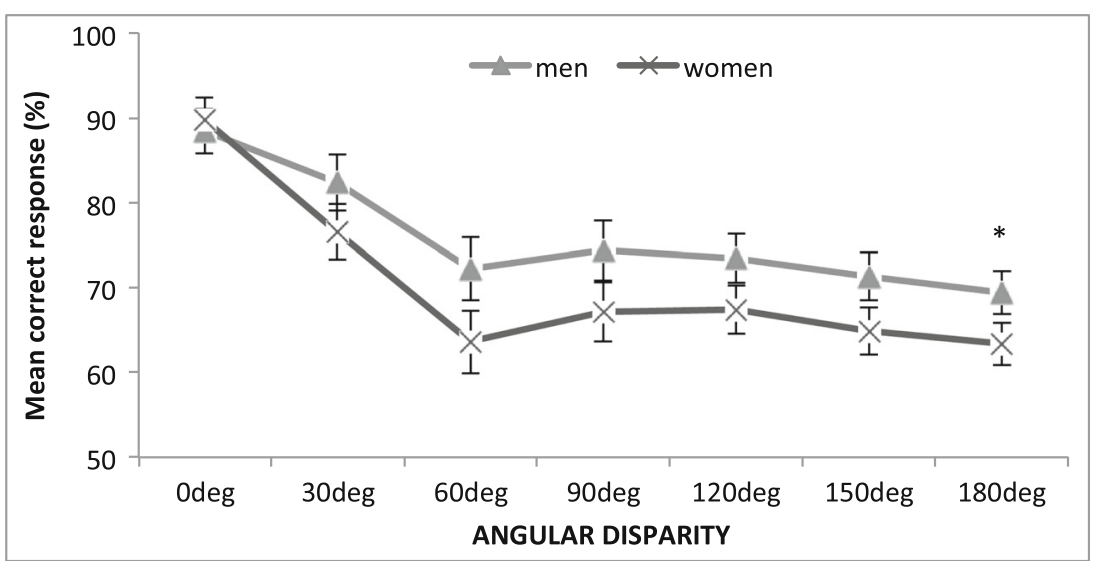

Fig. 9 Shows mean correct response for men and women at each degree of rotation. Note that $*$ shows the significance level at .05 level $(\mathrm{p}<.05)$

angular disparity between figures increased, consistent with Experiment 1 results. There was no overall sex difference, but mental rotation performance differed with greater $\mathrm{AD}$ with men's slight advantage in accuracy. Again, consistent with Experiment 1, we did not find robust sex differences.

Framing affected mental rotation performance; having a frame around the stimuli, overall, slowed rotation rate, regardless of the Frame Type. The Multiple Systems Framework (Zacks \& Michelon, 2005) may explain this effect of framing. Mental rotation involves object-based transformations. Adding a frame introduces a reference frame external to the object, potentially leading to conflicting mental rotation approaches. Another explanation involves a potential conflict in dimensionality between the frame (2-D) and the 3-D figures. The 2-D frame may serve as a flatness cue (Eby \& Braunstein, 1995), suggesting that people also think of the figures as 2-D (Reinhardt-Rutland, 2003). Inconsistencies interpreting represented dimensionality may hinder overall performance. Implications of the findings will be further discussed in the general discussion section.

Experiment 2 further explored individual differences in habitual spatial processing (Preference and Representation). Participants preferring verbal directions (compared to maps) had a stronger ADE with response time. This difference was further emphasized by frame type. The ADE separated for the different frame types (unframed, circular-, and rectangular) within participants with a verbal preference. This finding further supports the idea that how people habitually process

Table 2 Sex ratios in reference to habitual thinking styles (preference and representation)

\begin{tabular}{llllll}
\hline & \multicolumn{2}{l}{ Preference } & & & \multicolumn{2}{l}{ Representation } \\
\cline { 2 - 3 } \cline { 5 - 6 } & Verbal & Map & & Landmark & Survey \\
\hline Women & 9 & 26 & & 20 & 15 \\
Men & 4 & 14 & & 8 & 10 \\
\hline
\end{tabular}

spatial information reliably affects rotation performance, especially when introducing various types of stimuli.

\section{Experiment 3}

Three main modifications were done in Experiment 3. (1) We changed the dimensions of the surrounding box to further investigate the effects of framing. Since Experiment 2 results led us to suspect a possible frame-figure dimensionality mismatch, we replaced the structure around the stimuli with a 3-Dimensional box. (2) Whole and cut versions of mental rotation figures were again used to replicate Experiment 1 results to account for the strategy use. (3) There was an increase in the number of participants in order to ensure that habitual thinking categories (Representation and Sense of Direction) would be similarly represented across sexes.

\section{Method}

\section{Participants}

One hundred and ten WPI students (60 women and 50 men) participated for partial course credit. Participants were taking Introductory to Psychology as an elective course.

\section{Materials}

Mental rotation stimuli Three-dimensional block figures from Experiment 1 were again used. The Framed condition in Experiment 1 had 2-D rectangular shapes surrounding each figure. Here, 3-D prism boxes were created to replace 2-D boxes to frame the stimuli. Thus, the Framed condition in Experiment 2 had 3-D shapes surrounding each figure. Each box was approximately 189 (height) $\times 152$ (length) $\times 47$ (width) in size (see Fig. 12). The Unframed condition had the same stimuli as in Experiment 1 (see Fig. 12a, further 


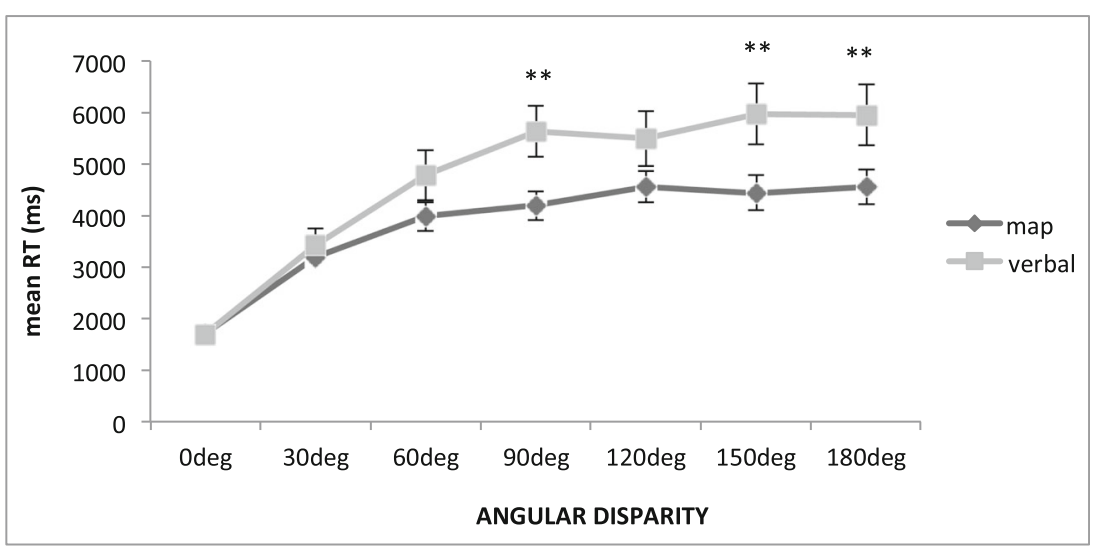

Fig. 10 Levels of preference over degrees of rotation for mean reaction time. Post-hoc analyses revealed significant results between map and verbal preference at each degree of rotation; * showing $\mathrm{p}<.05$ and $* *$ marking $\mathrm{p}<.01$

top left and Fig. 12b, top left). Stimuli pairs were presented as whole (Fig. 12a) and and cut (Fig. 12b) and paired to have the same (yet rotated) or different (mirror-reflections) combinations with a vertical line dividing the pair.

There were four blocks of trials: whole-unframed, wholeframed, cut-unframed, cut-framed. Each block started with 12 practice trials that were followed by 63 test trials. Within each block, participants were exposed to each rotation angle nine times, totaling to 36 times throughout the experiment. Overall, participants completed 280 trials excluding practice sessions. Of these test trials, 140 of them showed whole versions and 140 of them showed cut versions of these; 140 of them were unframed and 140 of them were framed; and 140 were the same pairs and 140 were different pairs.

Questionnaires Two questionnaires were used in Experiments 1 and 2: (1) the Individual Differences Questionnaire, which was the basis for Preference, and (2) the Mental Representation Questionnaire (Pazzaglia \& De Beni, 2001), which was used to categorize participants according to their Representation styles. In Experiment 3, the Individual Differences Questionnaire was replaced with a more comprehensive and widely used self-report on preference and sense-of-direction; the Sense of Direction (SOD). The Santa Barbara Sense-of-Direction Scale was used to assess environmental spatial ability and has been reported to correlate with objective spatial tasks (Hegarty, Richardson, Montello, Lovelace, \& Subbiah, 2002). It consists of two demographic questions on sex and age of the participant, followed by 15 positive and negative statements such as "I am very good at giving directions" and "I very easily get lost in a new city," respectively. Participants report their assessments for each statement on a Likert-type scale where 1 indicates strongly agree, and 7 indicates stongly disagree. As a result, each participant receives a computed score and a higher the score indicates a higher sense of direction (Hegarty et al., 2002).

\section{Design}

The present study had two independent variables: frame and figure consistency. For the frame variable participants saw block figures without a frame, and within a 3-D frame. For the figure consistency variable, they were exposed to whole stimuli and cut versions of them.

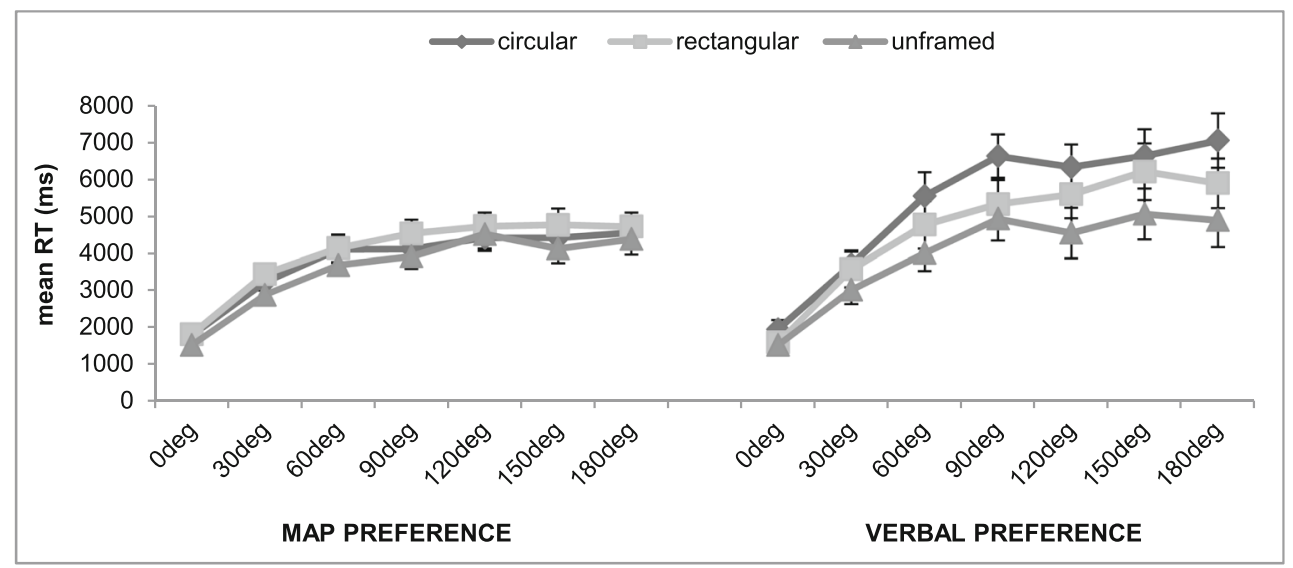

Fig. 11 Mental rotation performance of stimuli with different frame types over degrees of rotation as a function of preference. (a) (on the left) shows performance of map preference, and (b) (on the right) performance of verbal preference with mean reaction times 


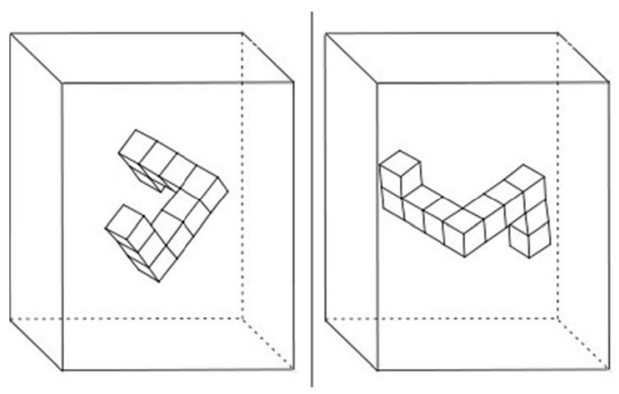

a
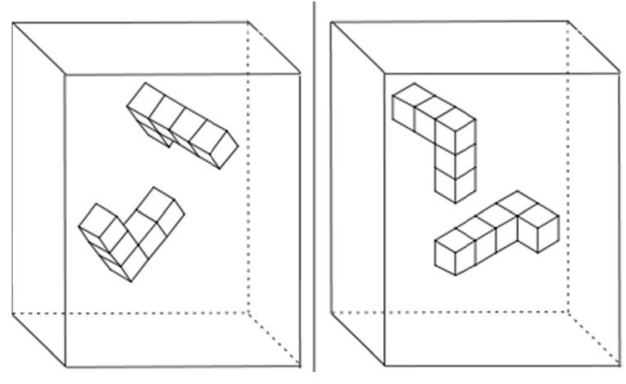

b

Fig. 12 (a) (left) A pair of whole-framed figures while (b) (right) shows a pair of cut-framed stimuli

Participants were categorized on sex, representation, and SOD according to their self-reports. Thus, the present study was based on a 2 (un-framed vs. framed, within participant) $\times 2$ (whole vs. cut, within participant) $\times 2$ (individual factors: sex, representation, or SOD, between participant) mixed design. The dependent variable was the performance on the Mental Rotation test.

\section{Procedure}

The procedure was very similar to Experiments 1 and 2 . The main distinction was the pre-screening. Participants completed the Representation Questionnaire prior to the experiment to ensure recruitment of roughly equal number of participants per category. When the participant came to the lab, he/she signed the consent form and completed the computerized Mental Rotation task via Inquisit. Instructions preceded each of the four experimental blocks, defined by stimuli conditions. They either saw a block of whole figures first, or a block of cut figures, and the order was counterbalanced across participants. A trial consisted of a fixation cross, presented for $500 \mathrm{~ms}$, followed by a pair of figures. Participants responded "same" or "different" by pressing designated keys, the placement of which was also counterbalanced. After completing the task, participants filled out the $S O D$ scale.

\section{Results}

\section{Coding and analysis}

Dependent measures included accuracy and reaction time (RT). Data from five participants were eliminated because they were outliers (two people) or had accuracy rates below $50 \%$ (three people). Participant's highest and lowest RTs at each angular disparity were trimmed because of high variability (Bush et al., 1993).

A mixed-design ANOVA was used with within-subject factors of Angular Disparity $\left(0^{\circ}, 30^{\circ}, 60^{\circ}, 90^{\circ}, 120^{\circ}, 150^{\circ}\right.$, and $180^{\circ}$ ), Figure Consistency (whole, cut) and Framing (unframed, framed) and between-participant factors: individual difference measures: Sex (men, women), Representation (survey, landmark), and SOD (high, low). Between-subject factors were analyzed separately by adding each, one by one, to the omnibus ANOVA.

Mauchly's test indicated that the assumption of sphericity was violated for Angular Disparity for RT data, $X^{2}(20)=182$, $p<.001$, and for the accuracy data, $X^{2}(20)=68.5, p<.001$. Therefore, degrees of freedom were corrected using Greenhouse-Geisser estimates of sphericity $(\varepsilon=.51)$ for RT, and Huynh-Feldt correction $(\varepsilon=.84)$ for accuracy. The assumption of sphericity has also been violated for Angular Disparity* Figure Consistency interaction; RT data, $X^{2}(20)$ $=35.2, p=.019$, and accuracy data, $X^{2}(20)=49.9$. On both RT and accuracy, degrees of freedom were corrected using Huynh-Feldt estimates of sphericity; $\varepsilon=.95$, and $\varepsilon=.91$, respectively.

\section{Mental rotation performance}

The mean RT for the experiment was 4,932 $\mathrm{ms}$ and the overall accuracy was $72.8 \%$. ADE with increased RT, $F(3.05,253)=$ $235, p<.001, \eta_{\mathrm{P}}^{2}=.74$, (Fig. 13a), and decreased accuracy, $F(5.05,551)=98.05, p<.001, \eta_{\mathrm{P}}{ }^{2}=.47$ (Fig. 13b) as a function of Angular Disparity.

\section{Do strategy-consistent-stimuli affect mental rotation performance?}

The analysis showed a main effect of Figure Consistency. Participants responded faster and more accurately to whole objects $\left(\mathrm{M}_{\mathrm{RT}}=4763 \mathrm{~ms}, \mathrm{M}_{\mathrm{acc}}=77.1 \%\right.$ accuracy $)$ than to cut ones $\left(\mathrm{M}_{\mathrm{RT}}=5101 \mathrm{~ms}, \mathrm{M}_{\mathrm{acc}}=68.6 \%\right), F(1,83)=4.49$, $p=.037, \eta_{\mathrm{P}}{ }^{2}=.051$ for $\mathrm{RT}$ and $F(1,109)=116, p<.001, \eta_{\mathrm{P}}{ }^{2}$ $=.52$ for accuracy.

Figure Consistency interacted with Angular Disparity with both RT, $F(5.70,473)=7.26, p<.001, \eta_{\mathrm{P}}^{2}=.080$, and accuracy, $F(5.48,597)=6.15, p<.001, \eta_{\mathrm{P}}^{2}=.053$. Participants consistently responded less accurately to cut figures for angular disparities and the performance difference for whole and cut figures was more evident at most degrees of rotation 

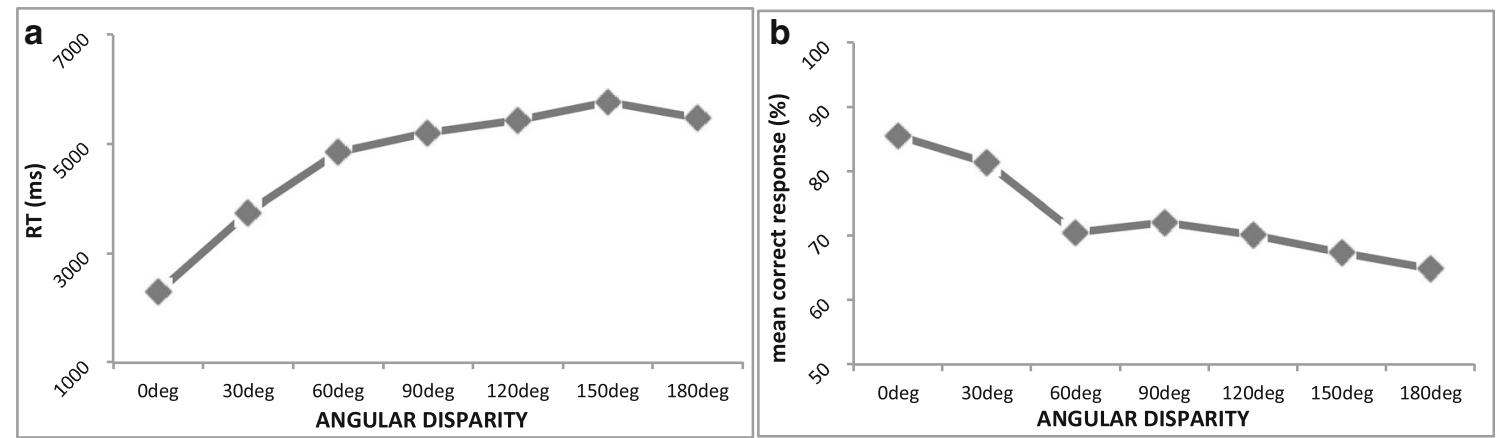

Fig. 13 Both reaction time (RT) (a; left) and accuracy (b; right) as a function of Angular Disparity. Note that the mean RTs are depicted on a 1,000$7,000 \mathrm{~ms}$ scale and mean correct response displays $50-100 \%$ scale to show the effect more clearly

(Fig. 14b). For RT data, participants rotated to whole and cut figures equally fast (Fig. 14a).

\section{Does framing stimuli affect mental rotation performance?}

Framing did not affect rotation performance by itself, $F(1,83)$ $=1.49, p=.23$, and it did not show any interactions with the other factors (all $F_{s}<1.0$ ).
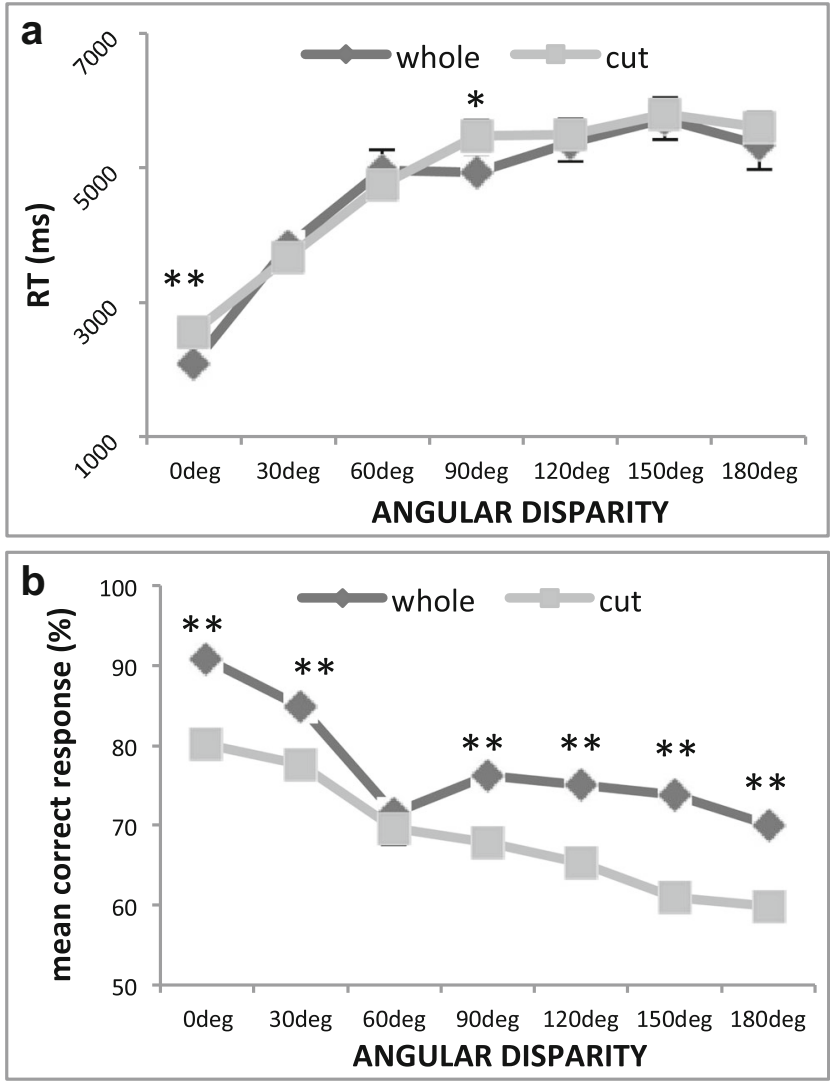

Fig. 14 Mean response times (a: top) and mean correct percentages (b: bottom) to whole and cut figures over degrees of rotation are depicted. Note the y axes for reaction time (RT) $(1,000-7,000 \mathrm{~ms})$ and accuracy $(50-100 \%)$ for a closer display. Post-hoc t-tests for both RT and accuracy data show significant results with asterisks. * alpha level of $.05(\mathrm{p}<.05)$; ** significance at the .01 level $(\mathrm{p}<.01)$

\section{Does sex of the participant affect mental rotation performance?}

Sex did not show a main effect for RT, $F(1,107)=1.63, p=$ .20 but men responded to 3-D figures more accurately $(\mathrm{M}=$ $75.8 \%), F(1,106)=7.41, p=.008, \eta_{\mathrm{P}}^{2}=.067$ than women $($ Macc $=70.5 \%)$. Sex did not show any further interactions with Angular Disparity or Figure Consistency, for either RT or accuracy data, (all $F_{S}<1.0$ ).

\section{Does habitual spatial thinking affect mental rotation performance?}

Mental representation (henceforth referred to as Representation) was categorized in similar numbers for each sex (see Table 3). Both women (57 \%) and men (56 \%) used slightly more landmark-centered representations, $\mathrm{c}^{2}(1, N=$ $110)=.005, p=.944$. It is important to note that both sexes were categorized in similar ratios in landmark and survey groups. Both sexes preferred map descriptions over verbal directions, $\mathrm{c}^{2}(1, N=110)=.535, p=.464$. Representation was not found to be significant as a main effect; either for RT data, $F(1,107)=.30, p=.58$ or for accuracy data, $F(1$, $108)=.31, p=.58$.

Sense of Direction (SOD) was categorized on participants' self-reports on rating their spatial information processing. The SOD score for each participant was computed and the ones above the mean were categorized as having high sense of direction and the ones scored below the mean were grouped as having low sense of direction. Fifty-one people scored as

Table 3 Sex ratios in reference to representation and sense of direction

\begin{tabular}{llllll}
\hline & \multicolumn{2}{l}{ Representation } & & \multicolumn{2}{l}{ Sense Of Direction } \\
\cline { 2 - 3 } \cline { 5 - 6 } & Landmark & Survey & & Low & High \\
\hline Women & 34 & 26 & & 30 & 30 \\
Men & 28 & 22 & & 29 & 21 \\
\hline
\end{tabular}


high SOD, and 59 people were categorized as low SOD (Table 3). Of those with high SOD, $58.8 \%$ were women and among the ones with low SOD, $50.8 \%$ were women. Both men and women were close in SOD distribution. There was no main effect of SOD, $F(1,107)=.050, p=.82$, among high and low SOD groups.

\section{Discussion}

Consistent with Experiments 1 and 2, the ADE effect was observed for both mental rotation rate and accuracy. As was found in Experiment 1, modifying 3-D stimuli to rotate whole and cut versions seemed to enforce the suggested strategy use. Participants were faster and more accurate in rotating whole figures as opposed to cut figures. More interestingly, the difference between whole and cut figure rotation was more evident for greater degrees of rotation, especially for accuracy data.

Unlike Experiments 1 and 2, however, framing with 3-D boxes did not affect the rotation performance. It might be because of the congruency between the dimensions of the frame and the figure. Consistent with previous research findings, participants did not have 2-D frames to serve as flatness cues (Eby \& Braunstein, 1995) or were forced to think the figures in 2-D to match the frame (Reinhardt-Rutland, 2003).

Replicating results found in Experiments 1 and 2, the only sex difference was found with men being more accurate than women. However, there was no difference in terms of rotation rate. Again, we can conclude that we did not find a robust sex difference, considering the modifications with the stimuli. Habitual spatial thinking styles (representation, and SOD) did not suggest any performance or strategy differences either and these will be further addressed in the general discussion.

\section{General discussion}

Many everyday activities engage mental rotation. Going back to Dave and Judy trying to place their sectional in their new living room, Dave might be rotating the couch as a whole, leading to a more effective decision about its placement. In contrast, Judy may have been mentally moving the individual parts. Having the walls of the room around the furniture, or interpreting the furniture as 3-D may have been another factor that affected the difference in their performance. The present research explored the contributions of stimulus differences and individual differences in habitual spatial processing to mental rotation performance.

It is important to note that our findings replicated the classic angular disparity effect (ADE; e.g., Blough \& Slavin, 1987; Collins \& Kimura, 1997; Cooper, 1975; Heil \& Jansen-Osmann, 2008; Kail et al., 1979;
Maccoby \& Jacklin, 1974; Shepard \& Metzler, 1971). ADE shows that rotation performance is directly affected by the angular difference between objects.

\section{Do stimuli characteristics affect mental rotation performance?}

Between Dave and Judy, would their rotation process be influenced by how sectional was brought in: one piece or within pieces? Would incorporating the walls of the room into this process help the task or disrupt it? In terms of stimulus differences, the holistic and piecemeal strategies suggest that people conceptualize the classic ShepardMetzler figures differently when engaging in mental rotation. The present research explored two different stimulus manipulations. First, we had whole and cut versions of the stimuli, predicting that cut figures would promote a piecemeal strategy. Second, we placed frames around individual stimuli within a pair. We had two different predictions for this manipulation (Experiments 1 and 3). The frame could emphasize the unity of the figure (Rock, 1990), leading to a more holistic approach or the frame could disrupt object-based reference frame (Zacks \& Michelon, 2005) use during rotation, thus impeding a holistic strategy.

Cutting the whole stimuli Results suggested that participants used mental rotation strategies in line with the stimulus consistency for 3-D figures. The rotation of cut figures was more consistent with a piecemeal strategy and rotation of whole figures with a holistic strategy. Participants more rapidly and accurately rotated whole compared to cut 3-D figures and this difference widened with increasing angular difference, as was shown in Experiment 1. This finding suggests participants used holistic rotation with whole figures and a piecemeal approach with cut figures. However, this finding was only evident when the frames were 2-D structures (Experiment 1) and did not replicate with 3-D frames (Experiment 3). In other words, 2-D structure around the figures emphasized the unitary configuration of the figures (Rock, 1990) engaged a more holistic strategy, whereas a 3-D frame did not affect or interfere with the use of strategies.

Framing the stimuli Our predictions for how framing would affect mental rotation were based on previous results within the literature. Framing did indeed interact with stimulus consistency and showed somewhat different findings across our studies. In Experiment 1, rotating 3-D figures within 2-D frames slowed cut figure rotation rate and more so with increased angular disparity, while no difference based on figure consistency emerged for unframed stimuli. In other words, results showed rotation strategies consistent with stimulus consistency (holistic for whole objects; piecemeal for cut 
objects) with framed 3-D stimuli. In Experiment 2, rotating 3$\mathrm{D}$ figures within circular or rectangular 2-D frames negatively affected rotation rate in comparison to unframed figures. Participants rotated figures within some kind of 2-D structure more slowly. In Experiment 3, however, there was no effect of frame. Taken all the findings into consideration, we can suggest that having a frame around the stimuli affects rotation, only if the frame dimension is incongruent with the target. When participants are faced with an incongruent mental rotation problem, they might need to change their perception of the figure itself because a 2-D frame suggests flatness (Eby \& Braunstein, 1995) and/or decreased depth (ReinhardtRutland, 2003).

\section{How do individual differences affect mental rotation performance?}

Sex has been the most studied individual difference with respect to mental rotation. We suggest that habitual spatial processing may help explain possible sex differences. Going back to the initial example, does Dave prefer maps and Judy prefer verbal directions? Does Dave usually form a more global, configural representation of larger environments while Judy thinks about the larger scale layouts in terms of its landmarks? If Dave and Judy differ in these ways, would they use different strategies for mental rotation? With a series of experiments, this study investigated how one's habitual thinking relates to his/her rotation strategy (Jordan et al., 2002; Kail et al., 1979; Thomsen et al., 2000). The habitual approaches we examined included preference for spatial information format (maps vs. verbal directions) and spatial representation approach (survey vs. landmark). Preference for maps and survey representation should both predict a more holistic, configural mental rotation strategy.

Sex Sex differences in the current study were not as robust as has been suggested in the literature (Collins \& Kimura, 1997; Kimura, 1999; Kail et al., 1979; Linn \& Petersen, 1985; Voyer et al., 1995). In all 3 experiments, men were more accurate in rotation than women. However, there were no additional effects. As hypothesized, sex may not be the only explanatory factor in assessing and understanding the difference in mental rotation performance.

Habitual spatial thinking Across the experiments, more women and men preferred maps over verbal directions while categorization across mental representations varied more. Still, those distributions show that sex is not the primary factor which determines one's preference regarding or mental representation of spatial information, or general sense of direction. We hypothesized that a Map Preference and a habitual Survey Representation should align with a holistic rotation strategy. People preferring maps rotated more accurately (Experiment 1) than those preferring verbal directions, and the difference was more pronounced with greater angular disparity (Experiments 1 and 2). Also in line with our hypothesis, people who habitually use a survey representation made more accurate and faster rotations (Experiment 1 ) than the ones taking a landmark approach (see also Pazzaglia and De Beni, 2006). This suggests that habitual spatial processing may help explain sex differences in mental rotation. To our knowledge this is the first finding linking habitual spatial thinking to rotation strategy use. However, these findings should be evaluated very carefully, because the results did not carry over to Experiment 3. One possible explanation was the difference in sample populations across experiments. Experiments 1 and 2 collected undergraduates from Tufts University. For Experiment 3 we recruited undergraduates at Worcester Polytechnic Institute (WPI), an institution attracting students with technical skills, preference, or inclinations. Research suggests that having superior spatial ability is correlated with a tendency towards Science-Technology-Engineering-Math (STEM) fields (Uttal \& Cohen, 2012) as well as success in these areas (Kozhevnikov, Motes, \& Hegarty, 2007; Vasilyeva \& Lourenco, 2012). Since we tested participants already studying in STEM fields, and since the students were roughly equally distributed across representation and SOD categories, possible effects due to habitual spatial processing might have been washed out.

\section{Conclusions}

Taken together the present studies extend extant findings on factors contributing to mental rotation strategy use in several ways. First, different features of the stimuli themselves play a role. In the current work this included figure consistency (whole vs. cut), presence of a frame around a stimulus, and, looking across studies, frame dimensionality. Second, how people habitually think about spatial information predicts mental rotation strategy use. Third, stimulus characteristics and individual differences in habitual spatial processing interact. In all cases, factors predicting or promoting a holistic strategy push strategy use in that direction and vice versa for the piecemeal strategy. This also suggests some cognitive flexibility in mental rotation strategy use.

The current research has implications for everyday life. We interact with maps or walk through rooms, buildings, or streets trying to create mental maps. Yet, we may reach a detour sign on a known route or have our global positioning (GPS) device fail. These situations would require us to mentally manipulate spatial information. Going back to the couple Dave and Judy, there might have been a difference between their rotation performances not merely 
because of their sex, but possibly because of some other factor that is perceived in the surrounding environment, the nature of the object they were rotating, or the way they have come to learn to process spatial information.

\section{References}

Alexander, G. M., \& Evardone, M. (2008). Blocks and bodies: Sex differences in a novel version of the mental rotations test. Hormones and Behavior, 53, 177-184.

Bauer, B., \& Jolicoeur, P. (1996). Stimulus dimensionality effects in mental rotation. Journal of Experimental Psychology: Human Perception and Performance, 22, 82-94.

Bethell-Fox, C. E., \& Shepard, R. N. (1988). Mental rotation: Effects of stimulus complexity and familiarity. Journal of Experimental Psychology: Human Perception and Performance, 14, 12-23.

Blough, P. M., \& Slavin, L. K. (1987). Reaction time assessments of gender differences in visual-spatial performance. Perception \& Psychophysics, 41, 276-281.

Borst, G., Kievit, R. A., Thompson, W. L., \& Kosslyn, S. M. (2011). Mental rotation is not easily cognitively penetrable. Journal of Cognitive Psychology, 23, 60-75.

Bryden, M. P., George, J., \& Inch, R. (1990). Sex differences and the role of figural complexity in determining the rate of mental rotation. Perceptual and Motor Skills, 70, 667-477.

Bush, L., Hess, U., \& Wolford, G. (1993). Transformations for withinsubject designs: A Monte Carlo investigation. Psychological Bulletin, 113, 566-579.

Butler, T., Imperato-McGinley, J., Pan, H., Voyer, D., Cordero, J., Zhu, Y. S., Stern, E., \& Silbersweig, D. (2006). Sex differences in mental rotation: Top-down versus bottom-up processing. Neuroimage, 32, $445-456$

Collins, D. W., \& Kimura, D. (1997). A large sex difference on a twodimensional mental rotation task. Behavioral Neuroscience, 111, 845-849.

Cooper, L. A. (1975). Mental rotation of random two-dimensional shapes. Cognitive Psychology, 7, 20-43.

Cooper, L. A., \& Shepard, R. N. (1973). Chronometric studies of the rotation of mental images. In W. G. Chase (Ed.), Visual information processing. New York: Academic Press.

Donders, F. C. (1969). On the speed of mental processes. Acta Psychologica, 30, 412-431.

Doyle, R. A., \& Voyer, D. (2013). Bodies and occlusion: Item types, cognitive processes, and gender differences in mental rotation. The Quarterly Journal of Experimental Psychology, 66, 801-815.

Doyle, R. A., Voyer, D., \& Lesmana, M. (2015). Item type, occlusion, and gender differences in mental rotation. The Quarterly Journal of Experimental Psychology, 1-15.

Eby, D. W., \& Braunstein, M. L. (1995). The perceptual flattening of three-dimensional scenes enclosed by a frame. Perception, 24, 981-993.

Freedman, R. J., \& Rovegno, L. (1981). Ocular dominance, cognitive strategy, and sex differences in spatial ability. Perceptual and Motor Skills, 52, 651-654

Folk, M. D., \& Luce, R. D. (1987). Effects of stimulus complexity on mental rotation rate of polygons. Journal of Experimental Psychology: Human Perception and Performance, 13, 395-404.

Gardony, A. L., Taylor, H. A., \& Brunye, T. T. (2014). What does physical rotation reveal about mental rotation? Psychological Science, $25,605-612$.
Geiser, C., Lehmann, W., \& Eid, M. (2006). Separating "rotators" from "nonrotators" in the mental rotations test: A multigroup latent class analysis. Multivariate Behavioral Research, 41, 261-293.

Gluck, J., \& Fitting, S. (2003). Spatial strategy selection: Interesting incremental information. International Journal of Testing, 3, 293-308.

Goldstein, D., Haldane, D., \& Mitchell, C. (1990). Sex differences in visual-spatial ability: The role of performance factors. Memory \& Cognition, 18, 546-550.

Halpern, D. F., Benbow, C. P., Geary, D. C., Gur, R. C., Hyde, J. S., \& Gernsbacher, M. A. (2007). The science of sex differences in science and mathematics. Psychological Science in the Public Interest, 8, 151 .

Halpern, D. F., \& Collaer, M. (2005). Sex differences in visuospatial abilities: More than meets the eye. In P. Shah \& A. Miyake (Eds.), Higher-level visuospatial thinking and cognition. Cambridge, MA: Cambridge University Press.

Hampson, E., Levy-Cooperman, N. A., \& Korman, J. M. (2014). Estradiol and mental rotation: Relation to dimensionality, difficulty, or angular disparity? Hormones and Behavior, 65, 238-248.

Hegarty, M., Richardson, A. E., Montello, D. R., Lovelace, K., \& Subbiah, I. (2002). Development of a self-report measure of environmental spatial ability. Intelligence, 30, 425-447.

Heil, M., \& Jansen-Osmann, P. (2008). Sex differences in mental rotation with polygons of different complexity: Do men utilize holistic processes whereas women prefer piecemeal ones? The Quarterly Journal of Experimental Psychology, 61, 683-689.

Hoyek, N., Collet, C., Fargier, P., \& Guillot, A. (2012). The use of the Vandenberg and Kuse mental rotation test in children. Journal of Individual Differences, 33, 62-67.

Hugdahl, K., Thomsen, T., \& Ersland, L. (2006). Sex differences in visuo-spatial processing: An fMRI study of mental rotation. Neuropsychologia, 44, 1575-1583.

Jansen-Osmann, P., \& Heil, M. (2007). The process of spatial knowledge acquisition in a square and a circular virtual environment. Advances in Cognitive Psychology, 3, 389-397.

Jordan, K., Schadow, J., Wuestenberg, T., Heinze, H.-J., Peters, M., \& Jancke, L. (2002). Women and men exhibit different cortical activation patterns during mental rotation tasks. Neuropsychologia, 40, 2397-2408.

Just, M. A., \& Carpenter, P. A. (1976). Eye fixations and cognitive processes. Cognitive Psychology, 8, 441-480.

Kail, R., Carter, P., \& Pellegrino, J. (1979). The locus of sex differences in spatial ability. Perception \& Psychophysics, 26, 182-186.

Kelly, J. W., McNamara, T. P., Bodenheimer, B., Carr, T. H., \& Rieser, J. J. (2008). The shape of human navigation: How environmental geometry is used in maintenance of spatial orientation. Cognition, 109, 281-286.

Kelly, J. W., \& McNamara, T. P. (2008). Spatial memories of virtual environments: How egocentric experience, intrinsic structure, and extrinsic structure interact. Psychonomic Bulletin \& Review, 15, 322-327.

Kimura, D. (1999). Sex and cognition. Cambridge, MA: MIT Press.

Khooshabeh, P., \& Hegarty, M. (2010). Inferring cross-sections: when internal visualizations are more important than properties of external visualizations. Human Computer Interaction, 25, 119-147.

Kozhevnikov, M., Motes, M. A., \& Hegarty, M. (2007). Spatial visualization in physics problem solving. Cognitive Science, 31, 549-579.

Lawton, C. A. (2010). Gender, spatial abilities, and wayfinding. In Handbook of gender research in psychology (pp. 317-341). New York: Springer.

Levinson, S. (1996). Frames of reference and Molyneux's question: Cross-linguistic evidence. In P. Bloom, M. A. Peterson, L. Nadel, \& M. F. Garrett (Eds.), Space and language (pp. 109-169). Cambridge: MIT Press. 
Linn, M. C., \& Petersen, A. C. (1985). Emergence and characterization of sex differences in spatial ability: A meta-analysis. Child Development, 56, 1479-1498.

Lippa, R. A., Collaer, M. L., \& Peters, M. (2010). Sex differences in mental rotation and line angle judgments are positively associated with gender equality and economic development across 53 nations. Archives of Sexual Behavior, 39, 990-997.

Lohman, D. F. (1986). The effect of speed-accuracy tradeoff on sex differences in mental rotation. Perception \& Psychophysics, 39, 427436.

Maccoby, E. E., \& Jacklin, C. N. (1974). The psychology of sex differences. Stanford, CA: Stanford University Press.

Masters, M. S., \& Sanders, B. (1993). Is the gender difference in mental rotation disappearing? Behavior Genetics, 23, 337-341.

Miller, D. I., \& Halpern, D. F. (2014). The new science of cognitive sex differences. Trends in Cognitive Sciences, 18, 37-45.

Moè, A. (2009). Are males always better than females in mental rotation? Exploring a gender belief explanation. Learning and Individual Differences, 19, 21-27.

Mou, W., Zhao, M., \& McNamara, T. P. (2007). Layout geometry in the selection of intrinsic frames of reference from multiple viewpoints. Journal of Experimental Psychology. Learning, Memory, and Cognition, 33, 145-154.

Mou, W., Zhang, H., \& McNamara, T. P. (2009). Novel-view scene recognition relies on identifying spatial reference directions. Cognition, $111,175-186$.

Naylor, S. J., Taylor, H. A., \& Cross, D. S. (1998). Gender and strategic processing differences in mental rotation. Unpublished manuscript.

Neuburger, S., Jansen, P., Heil, M., \& Quaiser-Pohl, C. (2012). A threat in the classroom: Gender stereotype activation and mental-rotation performance in elementary-school children. Zeitschrift für Psychologie Journal of Psychology, 220, 61-69.

Pazzaglia, F., \& De Beni, R. (2001). Strategies of processing spatial information in survey and landmark-centred individuals. European Journal of Cognitive Psychology, 13, 493-508.

Pazzaglia, F., \& De Beni, R. (2006). Are people with high and low mental representation abilities differently susceptible to the alignment effect? Perception, 35, 369-383.

Peters, M. (2005). Sex differences and the factor of time in solving Vandenberg and Kuse mental rotation problems. Brain and Cognition, 57, 176-184.

Pezaris, E., \& Casey, M. B. (1991). Girls who use "masculine" problemsolving strategies on a spatial task: Proposed genetic and environmental factors. Brain and Cognition, 171, 1-22.

Reilly, D., Neumann, D. L., \& Andrews, G. (2016). Sex and sex-role differences in specific cognitive abilities. Intelligence, 54, 147-158.

Reinhardt-Rutland, A. H. (2003). The framing effect diminishes pictorial depth perception: Implications for indirect manipulation via video links. In P. T. McCabe (Ed.), Contemporary ergonomics 2003 (pp. 177-182). Boca Raton: CRC.

Resnick, S. M. (1993). Sex differences in mental rotations: An effect of time limits? Brain and Cognition, 21, 71-79.

Richardson, J. T. E. (1994). Gender differences in mental rotation. Perceptual and Motor Skills, 78, 435-448.
Robert, M., \& Chevrier, E. (2003). Does men's advantage in mental rotation persist when real three-dimensional objects are either felt or seen? Memory \& Cognition, 31, 1136-1145.

Robertson, L. C., \& Palmer, S. E. (1983). Holistic processes in the perception and transformation of disoriented figures. Journal of Experimental Psychology: Human Perception and Performance, 9. 203-214.

Rock, I. (1990). The frame of reference. In I. Rock (Ed.), The legacy of Solomon Asch: Essays in cognition and social psychology (pp. 243268). Hillsdale, NJ: Lawrence Erlbaum.

Scali, R. M., Brownlow, S., \& Hicks, J. L. (2000). Gender differences in spatial task performance as a function of speed or accuracy orientation. Sex Roles, 43, 359-376.

Sharps, M. J., Price, J. L., \& Williams, J. K. (1994). Spatial cognition and gender instructional and stimulus influences on mental image rotation performance. Psychology of Women Quarterly, 183, 413-425.

Shepard, R. N., \& Metzler, J. (1971). Mental rotation of threedimensional objects. Science, 171, 701-703.

Strasser, I., Koller, I., Strauß, S., Csisinko, M., Kaufmann, H., \& Glück, J. (2010). Use of strategy in a 3-Dimensional spatial ability test. Journal of Individual Differences, 31, 74-77.

Thomsen, T., Hugdahl, K., Ersland, L., Barndon, R., Lundervold, A., \& Smievoll, A. (2000). Functional magnetic resonance imaging (fMRI) study of sex differences in a mental rotation task. Medical Science Monitor, 6, 1186-1196.

Uecker, A., \& Obrzut, J. E. (1993). Hemisphere and gender differences in mental rotation. Brain and Cognition, 22, 42-50.

Uttal, D. H., \& Cohen, C. A. (2012). Spatial thinking and STEM education: When, why and how. Psychology of Learning and Motivation, 57, 147-181.

Vandenberg, S., \& Kuse, A. R. (1978). Mental rotations: A group test of three dimensional spatial visualization. Perceptual and Motor Skills, 47, 599-604.

Vasilyeva, M., \& Lourenco, S. F. (2012). Development of spatial cognition. Wiley Interdisciplinary Reviews: Cognitive Science, 33, 349 362.

Voyer, D., \& Bryden, M. P. (1990). Gender, level of spatial ability, and lateralization of mental rotation. Brain and Cognition, 13, 18-29.

Voyer, D., \& Doyle, R. A. (2010). Item type and gender differences on the mental rotations test. Learning and Individual Differences, 20, 469472.

Voyer, D., \& Hou, J. (2006). Type of items and the magnitude of gender differences on the mental rotations test. Canadian Journal of Experimental Psychology/Revue Canadienne de Psychologie Expérimentale, 60, 91.

Voyer, D., Voyer, S., \& Bryden, M. P. (1995). Magnitude of sex differences in spatial abilities: A meta-analysis and consideration of critical variables. Psychological Bulletin, 117, 250-270.

Wang, R. F., \& Spelke, E. S. (2000). Updating egocentric representations in human navigation. Cognition, 77, 215-250.

Yuille, J. C., \& Steiger, J. H. (1982). Nonholistic processing in mental rotation: Some suggestive evidence. Perception \& Psychophysics, 31, 201-209.

Zacks, J. M., \& Michelon, P. (2005). Transformations of visuospatial images. Behavioral and Cognitive Neuroscience Reviews, 4, 96 118. 